\title{
Erratum
}

\section{Cumulative early childhood adversity and later antisocial behavior: The mediating role of passive avoidance - ERRATUM}

Idil Yazgan, Jamie L. Hanson, John E. Bates, Jennifer E. Lansford, Gregory S. Pettit and Kenneth A. Dodge

doi: https://doi.org/10.1017/S0954579419001809. Published online by Cambridge University Press, 23 March 2020.

Keywords: adverse childhood experiences, antisocial behavior, antisociality, early adversity, learning, passive avoidance

In the original version of Yazgan et al. 2021, there was an error in the numbering of the author affiliations. The correct affiliations are as follows:

Idil Yazgan ${ }^{1,2}$, Jamie L. Hanson ${ }^{3}$, John E. Bates ${ }^{4}$, Jennifer E. Lansford ${ }^{1}$, Gregory S. Pettit ${ }^{5}$ and Kenneth A. Dodge ${ }^{1}$

${ }^{1}$ Center for Child and Family Policy, Duke University, Durham, NC, USA; ${ }^{2}$ Department of Psychology, University of California,
San Francisco, San Francisco, CA, USA; ${ }^{3}$ Department of Psychology, University of Pittsburgh, Pittsburgh, PA, USA; ${ }^{4}$ Department of Psychological and Brain Sciences, Indiana University, Bloomington, IN, USA and ${ }^{5}$ Department of Human Development and Family Studies / College of Human Sciences, Auburn University, Auburn, AL, USA

The publisher apologizes for the error.

\section{Reference}

Yazgan, I., Hanson, J., Bates, J., Lansford, J., Pettit, G., \& Dodge, K. (2021). Cumulative early childhood adversity and later antisocial behavior: The mediating role of passive avoidance. Development and Psychopathology, 33 (1), 340-350. https://doi.org/10.1017/S0954579419001809
Cite this article: Yazgan I, Hanson JL, Bates JE, Lansford JE, Pettit GS, Dodge KA (2022). Cumulative early childhood adversity and later antisocial behavior: The mediating role of passive avoidance - ERRATUM. Development and Psychopathology 34: 1203, https:/ doi.org/10.1017/S0954579422000086

(c) The Author(s), 2022. Published by Cambridge University Press 